
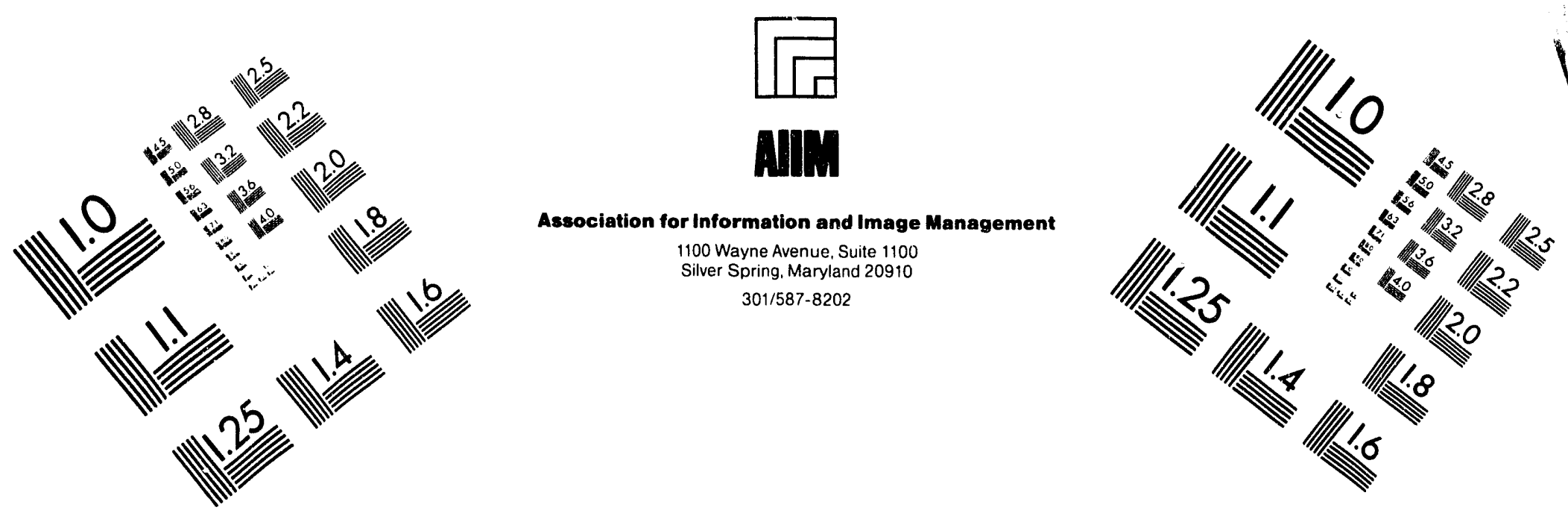

Centimeter

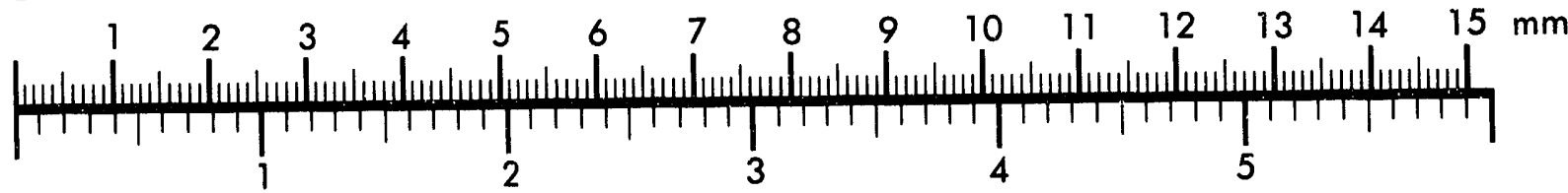
Inches
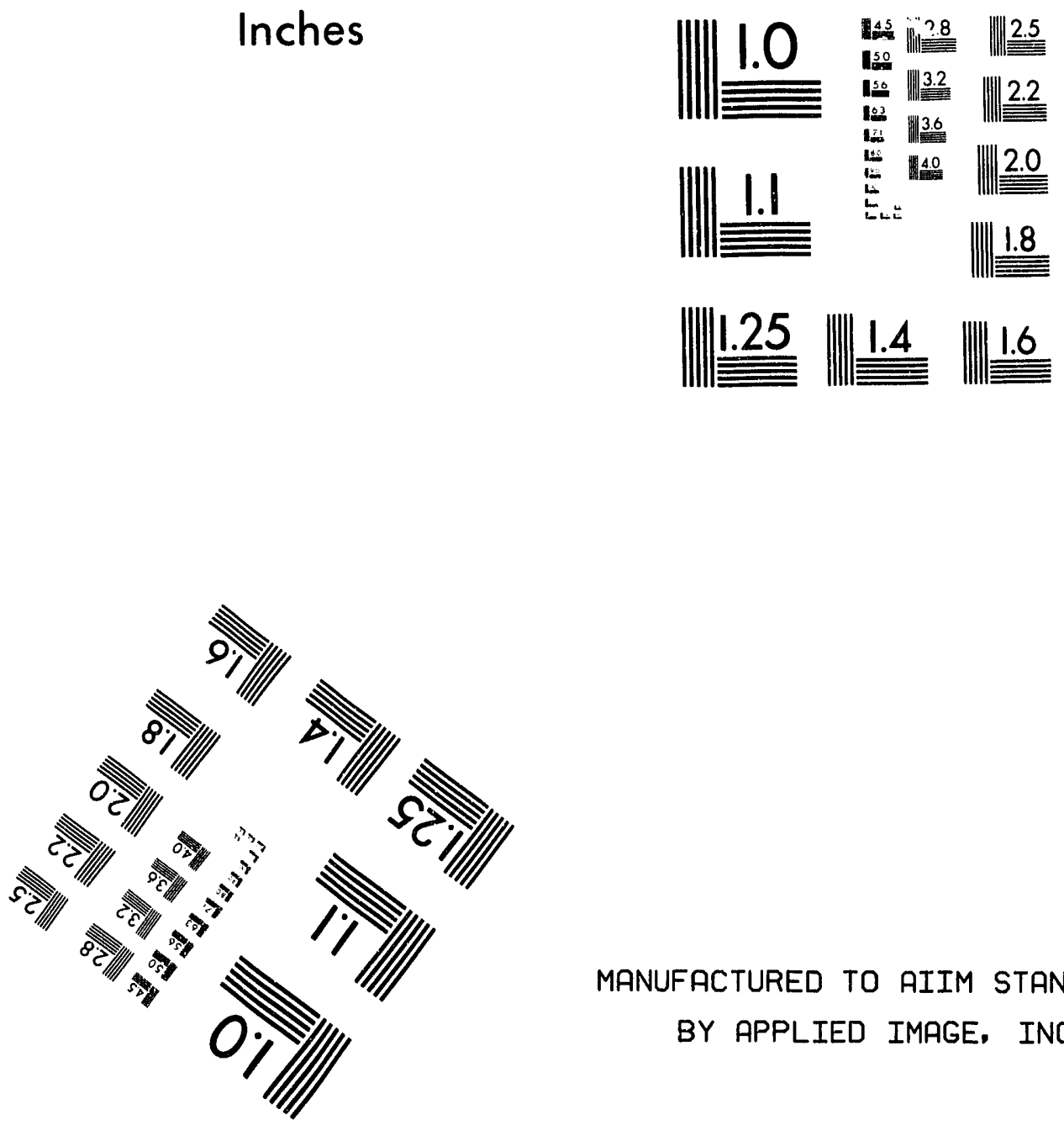

MANUFACTURED TO AIIM STANDARDS

BY APPLIED IMAGE, INC.

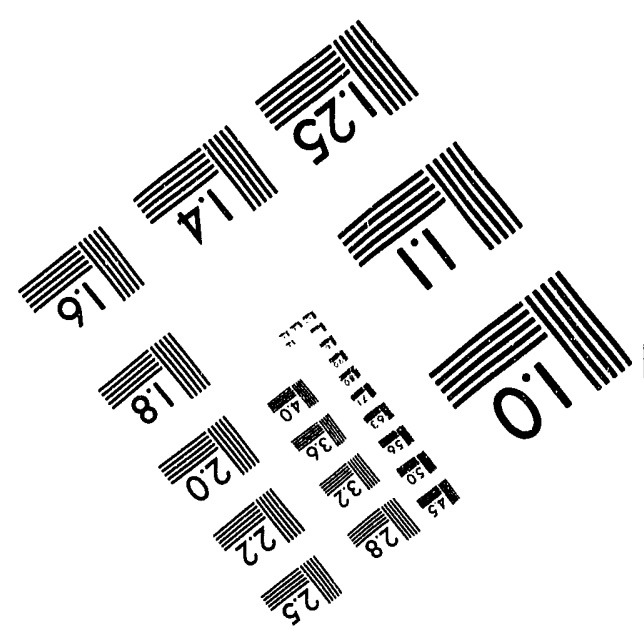



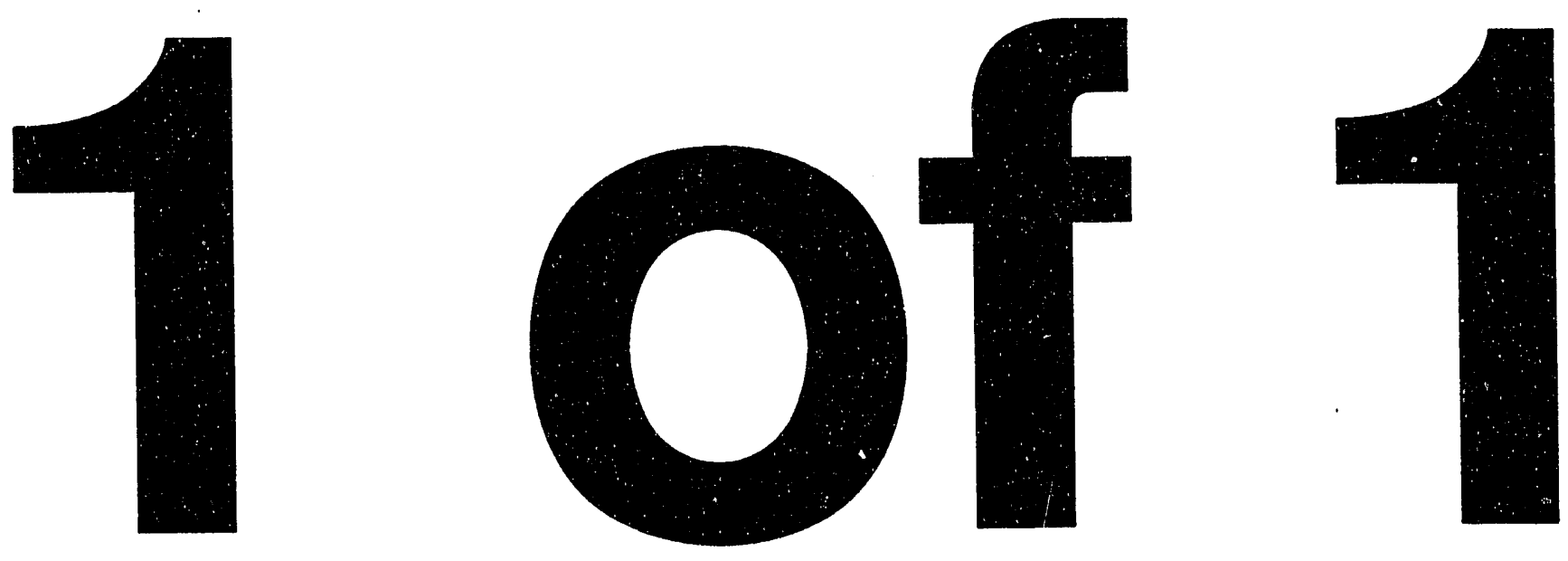
TITLE MOLECULAR COMPOSITES FROM. LIQUid CRYSTALLINE POLYMERS AND LIQUID CRYSTALLINE THERMOSETS

AUTHORISI Brian C. Benicewicz, MST-7

Elliot P. Douglas, MST-7

Rex P. Hjelm, Jr., LANSCE

SUBMITTED TO Proceedings of the International Workshop on Liquid

Crystalline Polymers, held June 1-4, 1993. Capri, ITALY

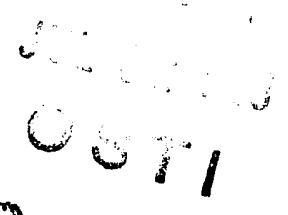

Ev accepiance of this article the oudisner recognizes inat ine US Government retains a nonexclusive rovalty-lree license to oublish or reproduce

ine oudisned torm o: inis conifibution or 10 allow olners to do so tor US Government Durdoses

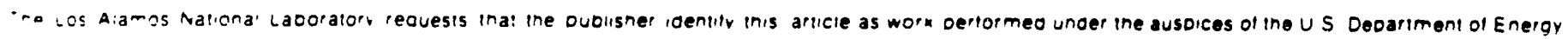






\title{
MOLECULAR COMPOSITES FROM LIQUIC CRYSTALLINE POLYMERS AND LIQUID CRYSTALLINE THERMOSETS
}

\author{
BRIAN C. BENICEWICZ and ELLIOT P. DOUGLAS \\ Materials Science and Technology Division \\ Los Alamos National Laboratory \\ Los Alamos, NM 87545, USA
}

and

REX P. HJELM, JR.

Los Alamos Neutron Scattering Center

Los Alamos National Laboratory

Los Alamos, NM 87545, USA

\begin{abstract}
We propose a new approach to molecular composites. This approach uses a mixture of a liquid crystalline polymer and a liquid crystalline thermoset to enhance the miscibility. Preliminary neutron scattering data is presented on a system of short and long rod aromatic amides. The data is interpreted using the interpenetrating phase model of Debye and Bueche. The analysis indicates that the scattering is consistent with this model and shows a characteristic length scale in the range of 70 to $80 \AA$. The intensity of the scattering is lower than calculated for the strong segregation limit, suggesting that there is some intermixing of the components.
\end{abstract}

\section{KEYWORDS}

Molecular composites; liquid crystalline polymers; liquid crystalline thermosets; neutron scattering; polymer blends; miscibility.

\section{INTRODUCTION}

There is a great deal of interest in the concept of molecular composites due to the large increases in mechanical properties which may be realized from this type of composite material (Hwang, et al., 1983). Fiber composite theory predicts that reinforcement of a matrix is controlled by the aspect ratio of the reinforcing element. Increasing the aspect ratio of the fiber is reported to increase tensile modulus by up to two orders of magnitude. Thus, the expected property improvements in molecular composites result from the reinforcement of a matrix at the molecular level by individually dispersed rigid rod polymer molecules. In addition, the formation of a molecular composite may alleviate the problem in conventional composites of failure at discrete phase separated fiber-matrix interfaces.

A recent review (Pawlikowski, et al., 1991) summarizes the current status of polymer molecular composites. Most of the work on molecular composites has focused on two reinforcelar composites. Most of the work on molecular composites has focther poly(p-phenylenebenzobisthiazole) or poly(benzimidazole). Thermoplastic and thermoset matrices have both been explored. In general, the major obstacle to overcome in the formation of a true molecular dispersion is the immiscibility of the rigid reinforcement and flexible matrix polymers. 
We have been exploring a new approach to molecular composites. This approach uses a mixture of a liquid crystalline polymer (LCP) reinforcement in a liquid crystalline thermoset (LCT) matrix. Composites made from LCP's and LCTs are expected to show enhanced miscibility as a consequence of both components' ability to form nematic liquid crystalline phases. Subsequent crosslinking of the thermoset should result in a homogeneous dispersion of rigid-rod polymer in a continuous three dimensional crosslinked network. We have previously reported on a large number of candidate LCT's, i.e., bifunctional rigid-rod molecules that form a nematic liquid crystalline phase and crosslink with retention of the nematic order. In this report, we describe our preliminary results on a mixture of a LCP and a LCT, shown in Fig. 1, using small angle neutron scattering (SANS) to determine the level of miscibility.

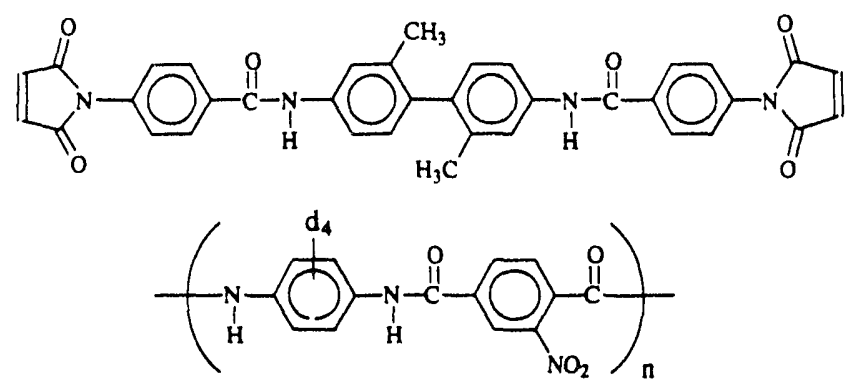

Fig. 1. Chemical structures of the LCT and deuterated LCP used in neutron scattering miscibility studies.

\section{EXPERIMENTAL}

The liquid crystalline thermoset, shown in Fig. 1, was synthesized as described previously (Hoyt and Benicewicz, 1990). The LCT used in this study was reported to form isotropic solutions in N-methyl-2-pyrrolidinone (NMP) up to a concentration of 30 weight percent. However, nematic textures were observed in films when $5 \%$ solutions were subjected to the drying conditions described below. The poly(p-phenylene-2-nitroterephthalamide) (PNTA) was prepared following the general phosphorylation procedure described by Higashi, et al. (1982). Deuterated p-phenylene diamine was obtained from MSD Isotopes and vacuum sublimed at $120^{\circ} \mathrm{C}$ directly before use in the polymerizations. Solution and film compositions are reported as weight/weight percent ratios. Solutions of the mixtures were made by dissolving the LCP and LCT in NMP at a total concentration of approximately $5 \%$. The isotropic solutions were cast onto glass plates and allowed to air dry for 24 hours, followed by oven drying at $60^{\circ} \mathrm{C}$. The films were then dried in vacuum at $150^{\circ} \mathrm{C}$ overnight.

Small-angle neutron scattering (SANS) measurements were carried out on the small-angle diffractometer (SAD) at the Intense Pulsed Neutron Source (IPNS) at Argonne National Laboratory. Data was reduced to differential scattering cross section per unit volume, $\mathrm{d} \Sigma(Q) / \mathrm{d} \Omega$, ( $\Sigma$ is the cross section per unit volume and $\Omega$ is solid angle in steradians) as a function of momentum transfer, Q, using methods described previously (Hjelm, 1988; Hjelm and Seeger, 1990; Seeger and Hjelm, 1991). $Q$ is related to the neutron wavelength, $\lambda$, and scattering angle, $2 \theta$, as $Q=2 \pi / \lambda \sin \theta$. 


\section{RESULTS}

Film samples considered here were produced by drying solutions of LCP mixed with LCT in weight proportion $60 / 40$ from the isotropic solution in NMP at $60^{\circ} \mathrm{C}$, as described in the methods section. Four such films were considered, each differing by the amount of $d_{4}$-LCP (Fig. 1). In this case there were $0,0.50,0.75$ and 1.0 weight fraction of deuterated yerses nondeuterated material. Figure 2 shows the results of the SANS measurements. There are two observations from the data shown in Fig 2. First is that there are neutron scattering density fluctuations on length scales probed by the small-angle scattering measurement, namely 10 $200 \mathrm{~A}$. Second is that the observed neutron scattering is dependent on the fraction of deuterated LCP in the sample.

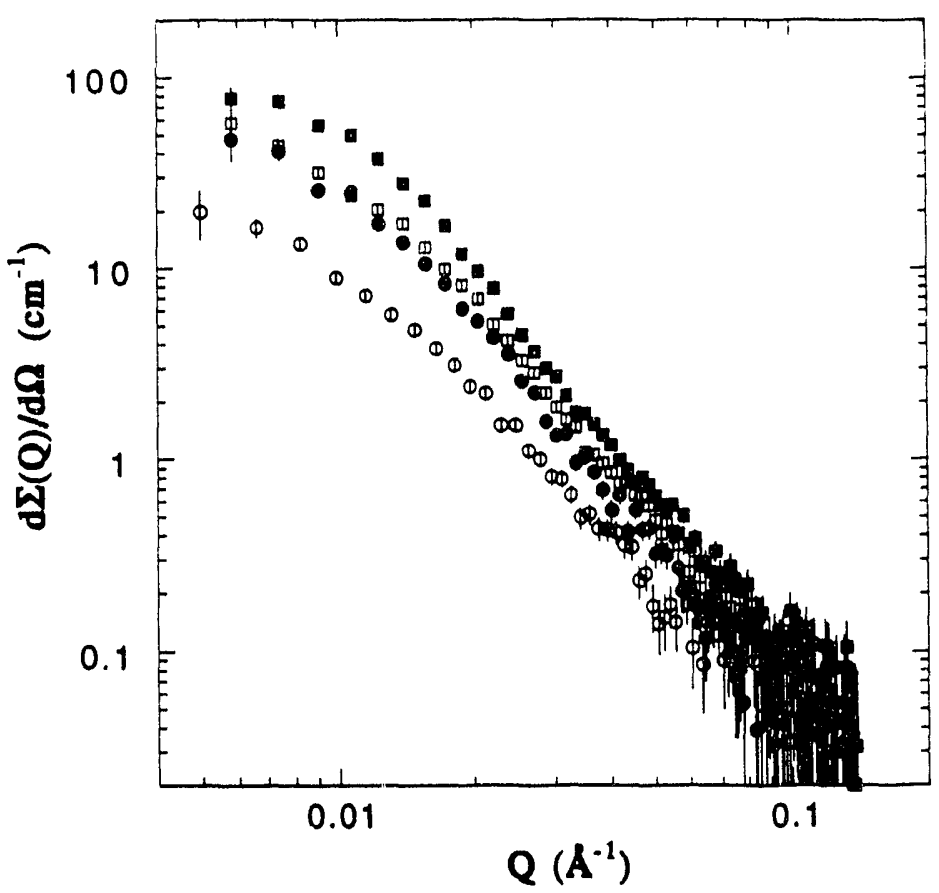

Fig. 2. Small-angle neutron scattering of LCP-LCT composite mixtures: mixtures are $60 \%$ LCP and $40 \%$ LCT by weight. Samples differ in fraction of LCP that is deuterated. $O, f_{d-L C P}=0 ; 0, f_{d-L C P}=0.5$; 口. $f_{d-L C P}=0.75 ; \square, f_{d-L C P}=1.0$.

We interpret these data using the model of Debye and Bueche (1949), which was originally used to describe inhomogeneities in acrylic resins. This model is characterized as a pair of randomly interpenetrating phases having different scattering length densities. Debye et al. (1949; 1957) showed that the correlations in scattering length density fluctuations betwee... two points separated by a distance, $r$, in the sample is given by,

$$
p(r)=4 \pi r^{2} \exp (-r / \zeta)
$$


where $\zeta$ is a characteristic distance of the exponential fall off of the correlation function, $p(r)$. Here we have assumed that the sample is isotropic. The scattering expected for this distribution is given by

$$
\mathrm{d} \Sigma(\mathrm{Q}) / \mathrm{d} \Omega=\frac{\mathrm{d} \Sigma(0) / \mathrm{d} \Omega}{\left(1+\mathrm{Q}^{2} \zeta^{2}\right)^{2}}
$$

Here, $d \Sigma(0) / d \Omega$ is the differential scattering cross section per unit volume at $Q=0$. If a straight line results by plotting $[\mathrm{d} \Sigma(\mathrm{Q}) / \mathrm{d} \Omega]^{-/ 2}$ verses $\mathrm{Q}^{2}$ then the scattering is consistent with this model. In this case the intercept is interpreted as $[\mathrm{d} \Sigma(0) / \mathrm{d} \Omega]^{-1 / 2}$ and the slope as $[\mathrm{d} \Sigma(0) / \mathrm{d} \Omega]^{-1 / 2} \zeta^{2}$. The SANS data plotted using the Debye-Bueche analysis is shown in Fig. 3 , from which we see that a straight line is a good approximation to the data. At this stage we have not exhausted all other possible models in fitting the data. Thus the analysis must be considered preliminary as to the details of phase morphology. Regardless, the general conclusions drawn here transcend the details of the model.

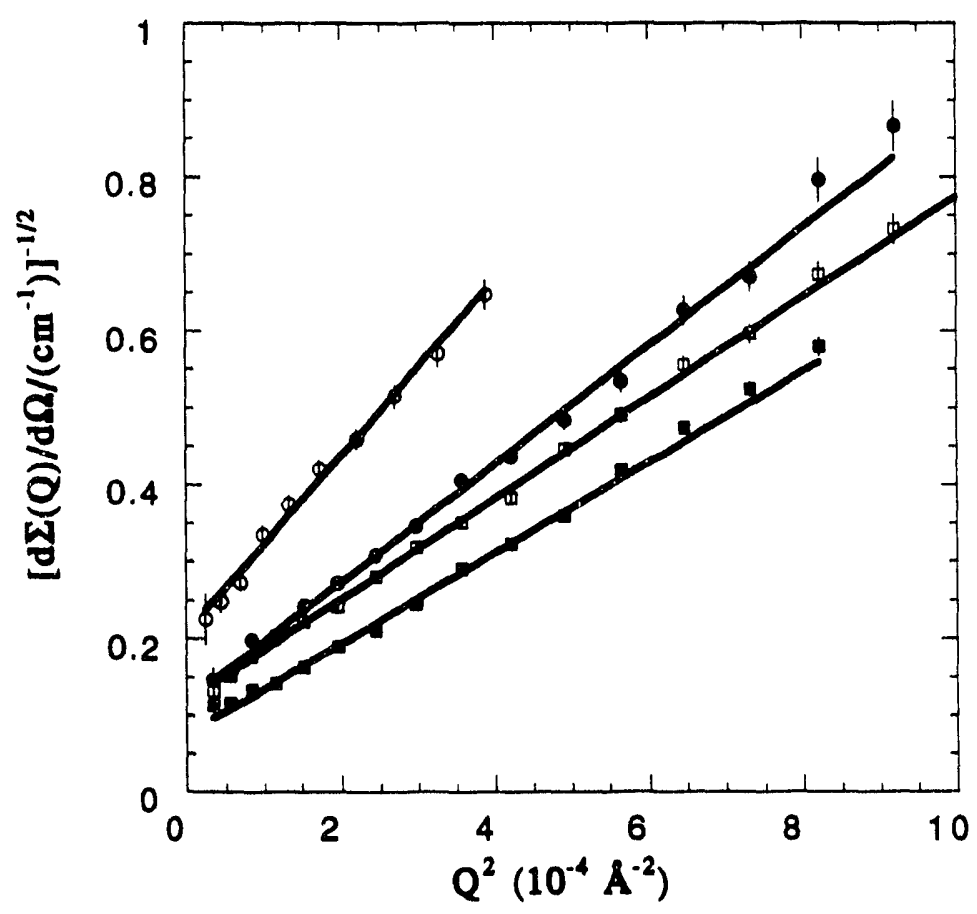

Fig. 3. Debye-B ueche analysis of small-angle neutron scattering data: Analysis of data shown in Fig. 3. $0, f_{d-L C P}=0 ; 0, f_{d-L C P}=0.5$; $\square, f_{d-L C P}=0.75 ; \square, f_{d-L C P}=1.0$. 
Table I

Parameters from the Debye-Bueche analysis

\begin{tabular}{ccc}
\hline \hline $\mathrm{f}_{\mathrm{d}-\mathrm{LCP}{ }^{\mathrm{a}}}$ & $\mathrm{d} \Sigma(0) / \mathrm{d} \Omega\left(\mathrm{cm}^{-1}\right)^{\mathrm{b}}$ & $\xi(\AA)^{\mathrm{b}}$ \\
\hline 0.0 & $23(1)$ & $74(2)$ \\
0.50 & $70(5)$ & $80(2)$ \\
0.75 & $69(3)$ & $74(1)$ \\
1.0 & $173(14)$ & $88(2)$ \\
\hline \hline
\end{tabular}

a Weight fraction of total PNTA that is deuterated.

Number in parentheses are root mean squared deviations from the regression.

Table I summarizes the $\mathrm{d} \Sigma(0) / \mathrm{d} \Omega$ and $\zeta$ parameters derived from the Debye-Bueche analysis of Fig. 3. $d \Sigma(0) / d \Omega$ varies with the fraction of $d_{4}-$ PNTA, $f_{\mathrm{d}-L C P}$. The characteristic correlation distance, $\zeta$, is in the range of $70-80 \AA$.

Scattering derives from the fluctuations of scattering length density, $\rho(R)$, from an average value, $\bar{\rho}$, at every point, $R$, within the sample. If we calculate the square of this fluctuation as $\Delta p(R)^{2}=(\rho(R)-\rho)^{2}$, then

$$
\frac{d \Sigma(Q)}{d \Omega}=\left\langle\Delta p^{2}\right\rangle \int_{0}^{\infty} p(r) \frac{\sin Q r}{Q r} d r
$$

where the angle brackets indicate an average over the entire sample. Debye, et al. (1957) showed that for random interpenetrating phases

$$
\left\langle\Delta \varphi^{2}\right\rangle=\Delta \rho_{\mathrm{ab}}^{2} \Phi(1-\Phi)
$$

where $\Phi$ is the volume fraction of one of the two phases; $\Delta p_{\mathrm{ab}}$ is the difference in scattering length density between the two phases and is given as

$$
\Delta p_{\mathrm{ab}}^{2}=\left(\phi_{\mathrm{a}}-\phi_{\mathrm{b}}\right)^{2}\left(f_{\mathrm{d}-L C P}^{2} \Delta \phi_{\mathrm{dh}}^{2}+2 f_{\mathrm{d}-L C \mathrm{P}} \Delta p_{\mathrm{dh}} \Delta p_{\mathrm{PT}}+\Delta p_{\mathrm{PT}}^{2}\right)
$$

Here $\phi_{a}$ and $\phi_{b}$ are the volume fractions of LCP in, respectively, phase a (the LCP-rich phase) and $b$ (the LCT-rich phase); $\Delta \rho_{\mathrm{dh}}$ is the difference between the scattering length densities of the deuterated and protonated LCP's; and $\triangle p_{P T}$ is the difference in between the scattering length densities of the protonated LCP and LCT.

It is easy to show from equations [1], [3] and [4] that

$$
\frac{\mathrm{d} \Sigma(0)}{\mathrm{d} \boldsymbol{\Omega}}=8 \pi \zeta^{3} \Delta p_{\mathrm{ab}}^{2} \Phi(1-\Phi)
$$


From this and equation [5] we see that a plot of $\mathrm{d} \Sigma(\mathrm{Q}) / \mathrm{d} \Omega \zeta{ }_{-}{ }^{3}$ verses $f_{\mathrm{d}-\mathrm{LCP}}$ will give a parabola, assuming that the volume fraction of each phase, $\Phi$, does not change with $f_{\text {d.LCP. }}$. A plot for this data is given in Fig. 4 and the regression parameters are given in Table fil. The values for $\Phi(1-\Phi)\left(\Phi_{a}-\Phi_{b}\right)^{2}$ are calculated by equating the regression parameters with the coefficients for like powers in $f_{d-L C P}$ in equation [6] (when we replace equation [5] for $\Delta p_{a b}$ ). We observe no significant differences in these values, with 0.035 being the most likely. In the completely phase separated case $\Phi=0.59, \phi_{\mathrm{a}}=1$ and $\phi_{\mathrm{b}}=0$, in which case $\Phi(1-\Phi)\left(\phi_{\mathrm{a}}-\Phi_{\mathrm{b}}\right)^{2}$ $=0.24$. nearly an order of magnitude higher than the experimental values. This result strongly suggests that there is not strong segregation of the LCP and LCT in this system. Rather, there appears to be some partial mixing, with each phase being rich in one component but having substantial amounts of the minor component also present.

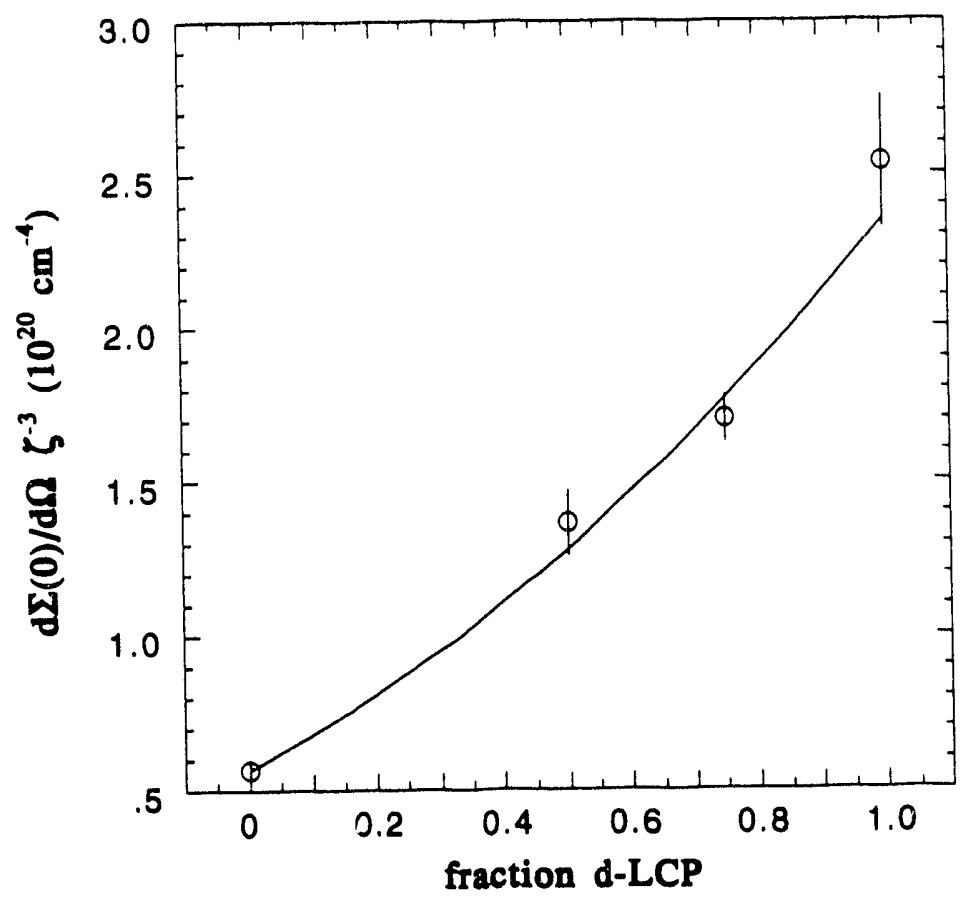

Fig. 4. Dependence of $\mathrm{d} \Sigma(0) / \mathrm{d} \Omega \zeta^{3}$ on the fraction of deuterated LCP: $O$, Values computed from the data given in Table III; - leastsquares regression of the data as discussed in the text.

\section{DISCUSSION}

The two important conclusions derived from this study are that there is phase separation in this system on the scale of $70-80 \AA$, and that each phase is not composed of the pure components, but rather is a mi-ture of LCP and LCT. The first conclusion is derived from the shape of the scattering curves in Fig. 2. The second results from the observation that the scattering intensity is far lower than expected for a strongly segregated microphase separated system.

To understand some uspects of this behavior we consider the basic theory of Abe and Flory (1978). This theory predicts the phase diagram for the mixing of long and short rod-like 
Table II

Calculated scattering-length densities of LCP-LCT

\begin{tabular}{ccc}
\hline \hline Component & Density $\left(\mathrm{cm}^{3} \mathrm{~g}^{-1}\right)^{\mathrm{a}}$ & $\rho_{\mathrm{p}}\left(10^{10} \mathrm{~cm}^{-2}\right)^{\mathrm{b}}$ \\
\hline $\mathrm{d}_{4}$-PNTA & 1.47 & 5.1 \\
PNTA & 1.47 & 3.8 \\
LCT & 1.43 & 3.0 \\
NMP & 1.033 & 0.9 \\
\hline \hline
\end{tabular}

Density determined by picnometry. Total error about $5 \%$.

Scattering length density, $\rho_{p}=(\rho / W) \Sigma n_{i} b_{i}$, where $W$ is the molecular weight, $n_{i}$ and $b_{i}$ are respectively the number and neutron scattering length of the $\mathrm{i}^{\mathrm{th}}$, atomic species in the molecule.

Table III
Parameters of fitting the dependence of $d \Sigma(0) / d \Omega \zeta^{-3}$ on $f_{d-L C P}$ According to Equation [6]

\begin{tabular}{ccc}
\hline \hline Term & $\begin{array}{c}\text { Regression Parameter } \\
\left(10^{-4} \mathrm{~cm}^{-4}\right)\end{array}$ & $\Phi(1-\Phi)\left(\phi_{\mathrm{a}}-\phi_{\mathrm{b}}\right)^{2}$ \\
\hline $8 \pi \Phi(1-\Phi)\left(\phi_{\mathrm{a}}-\phi_{\mathrm{b}}\right)^{2} \Delta \phi_{\mathrm{PT}}{ }^{2}$ & $0.57(0.04)$ & $0.035(0.002)$ \\
$16 \pi \Phi(1-\Phi)\left(\phi_{\mathrm{a}}-\phi_{\mathrm{b}}\right)^{2} \Delta \phi_{\mathrm{dh}} \Delta \phi_{\mathrm{PT}}$ & $1.1(0.7)$ & $0.02(0.01)$ \\
$8 \pi \Phi(1-\Phi)\left(\phi_{\mathrm{a}}-\phi_{\mathrm{b}}\right)^{2} \Delta \phi_{\mathrm{dh}}{ }^{2}$ & $0.7(0.8)$ & $0.02(0.02)$ \\
\hline
\end{tabular}

molecules. The treatment considers only the entropy of packing long rods and short rods in the presence of a small solvent species. The predicted phase behaviec is complex, characterized by isotropic mixing at dilute rod concentrations and biphase behavior at higher concentrations. The long rods readily form a nematic phase at sufficiently high concentrations, and the biphase behavior is a consequence of exclusion of the shorter rods from this phase. The effects are entirely entropic in origin, as the rods are considered to be non-interacting, and further, there is no interaction with the solvent.

Our results show that this description is far too simple to describe the detailed behavior of this system, and clearly further theoretical work is needed to give a more complete description. The most important factor is the enthalpy of interaction between the short LCT and long LCP molecules. There should be strong attractive interactions between these molecules due to molecules. There should be strong attractive interactions between these molecules due to hydrogen bonding and London dispersion forces, as both components are aromatic amides. In addition, there is the effect of the solvent, NMP, which must be considerable, as it is a good solvent for both components. Both of these factors are anticipated to improve mixing, working against the entropic effects described by Abe and Flory (1978) that tend to separate the components. Another factor is that PNTA is a semi-flexible molecule, not a rigid rod, and this can also affect the behavior. In addition, there are kinetic effects associated with the removal of solvent and the evolution of the structures as the liquid approaches the gel point in the drying film. Hence, it is likely that the structure observed in the films reflects closely the structure present at the gel composition point on the phase map.

A more complete understanding of the parameters that are important in realizing a true molecular composite will be important in future work. Clearly we have demonstrated that this system, though not a complete realization of this ideal, approaches it at least as closely as has been demonstrated previously for the best mixtures, (Pawlikowski, et al., 1991) even without 
optimizing our system. It is likely that other formulations and sample preparations will have the components dispersed to a greater or lesser degree than observed in this study. An understanding of the basic thermodynamic and kinetic factors which affect molecular-level mixing will help us to determine the appropriate conditions for the formation of a true molecular composite. Further, the measurement of mechanical properties will test the theoretical notions of reinforcement which is driving work in these materials.

\section{ACKNOWLEDGMENTS}

This work was supported by Laboratory Directed Research and Development Funding of the Los Alamos National Laboratory which is supported by the United States Department of Energy under contract W-7405-ENG-36 to the University of California. This work benefited from the use of the Small-Angle Neutron Diffractometer at IPNS , Argonne National Laboratory which is supported by the United States Department of Energy, Office of Basic Energy Sciences and other Department of Energy Programs under contract W-31-109-ENG38 to the University of Chicago. We thank Dr. P. Thiyagarajan (IPNS) and David Langlois (MST-7, LANL) for help in carrying out the measurements.

\section{REFERENCES}

Abe, A. and P.J. Flory (1978). Statistical thermodynamics of mixtures of rodlike particles. 2. Ternary systems. Macromolecules, $11,1122-1126$

Debye, P. and A.M. Bueche (1949). Scattering by an inhomogeneous solid. J. Appl. Phys., 20, 518-525.

Debye, P., R. Anderson and H. Brumberger (1957). Scattering by an inhomogeneous solid. II. The correlation function and its application. J. Appl. Phys., 28, 679-683.

Hjelm, R.P. (1988). Resolution of time-of-flight low-Q diffractometers: instrumental, data acquisition and reduction factors. J. Appl. Cryst., 21, 618-628.

Hjelm, R.P. and P.A. Seeger (1989). Sime-of-flight small-angle neutron scattering data reduction and analysis at LANSCE with program SMR. Conferences in Physics, 97, 367-387.

Higashi, F., S. Ogata and Y. Aoki (1982). High-molecular weight poly(p-phenyleneterephthalamide) by the direct polycondensation reaction with triphenyl phosphite. $J$. Polym. Sci.: Polym. Chem. Ed., 20, 2081-2087.

Hoyt, A.E. and B.C. Benicewicz (1990). Rigid rod molecules as liquid crystal thermosets. I. Rigid rod amides. J. Polym. Sci.: Part A: Polym. Chem., 28, 3403-3415.

Hwang, W.-F., D.R. Wiff, C.L. Benner and T.E. Helminiak (1983). Composites on a molecular level: Phase relationships, processing, and properties. J. Macromol. Sci. - Phys., B22(2), 231-257.

Pawlikowski, G.T., D. Dutta and R.A. Weiss (199!). Molecular composites and self-reinforced liquid crystalline polymer blends. Annu. Rev. Mater. Sci., 21, 159-84.

Seeger, P.A. and R.P. Hjelm (1991). Small-angle neutron scattering at pulsed spallation sources. J. Appl. Cryst., 24, 467-478. 

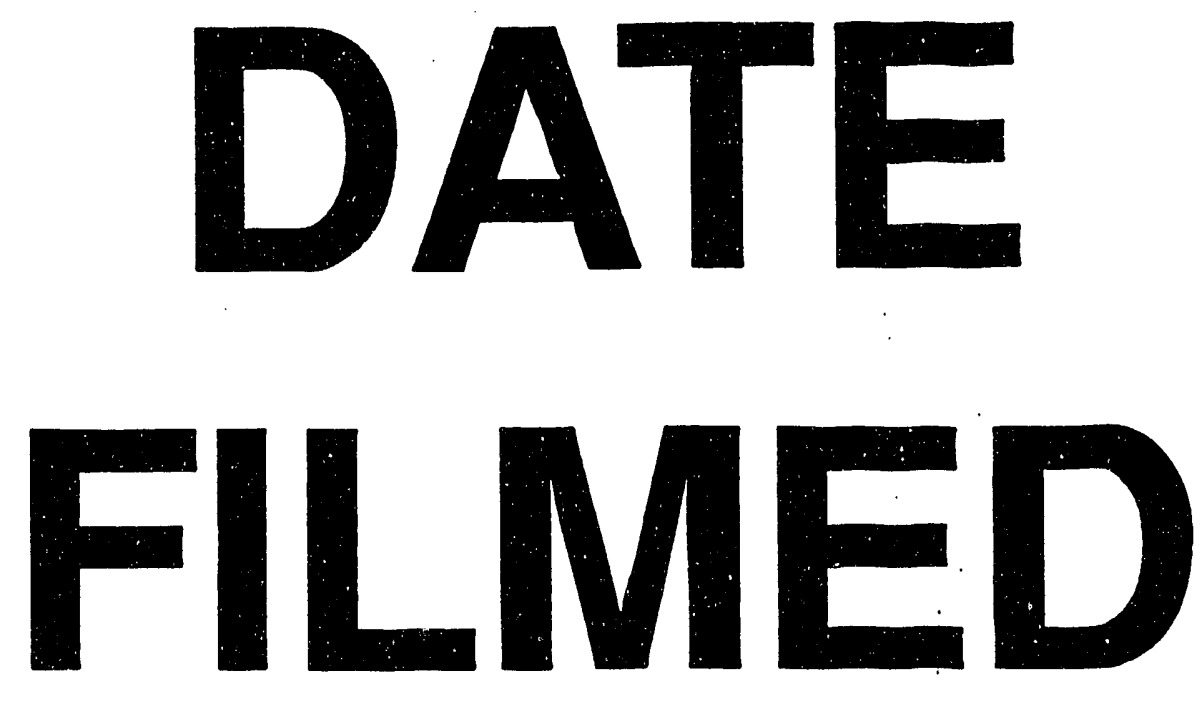

$9 / 16 / 93$
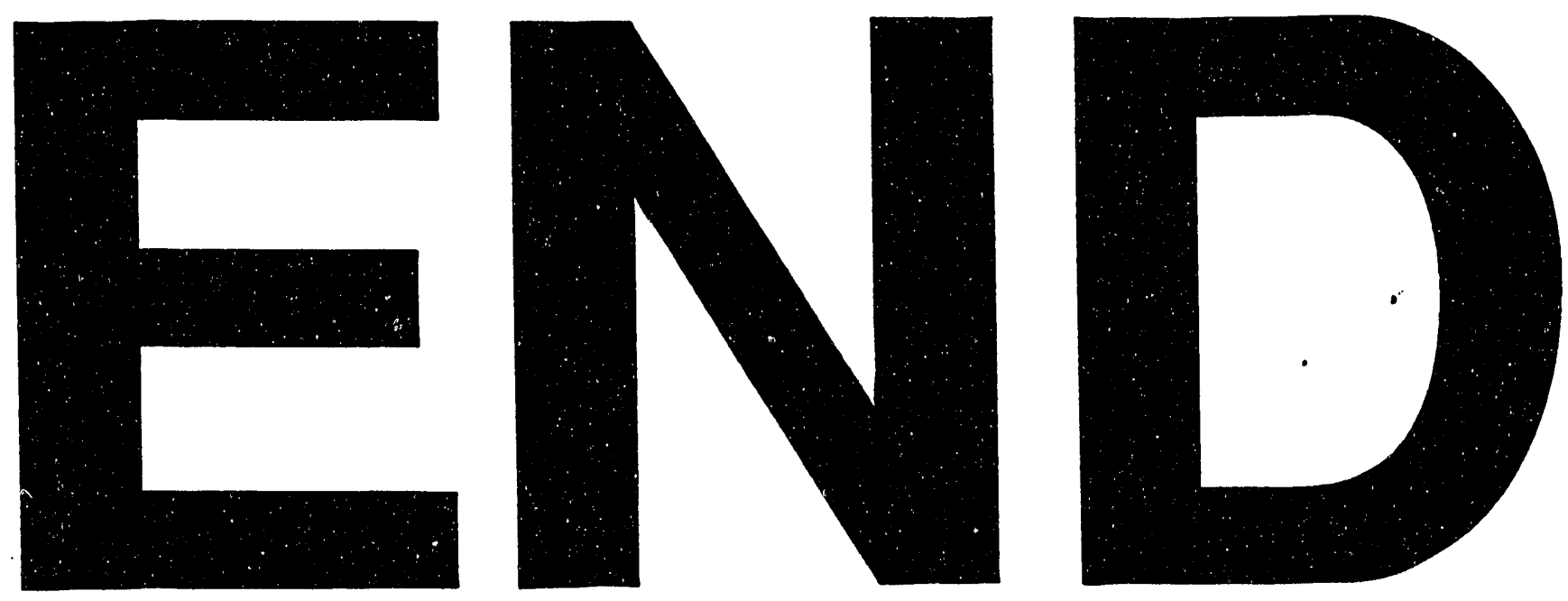
